Second order Lagrange multiplier approximation for constrained shape optimization problems

K. Eppler and H. Harbrecht ${ }^{1}$

1 TU Chemnitz, Faculty of mathematics

Preprint 2003/35

Preprint-Reihe des Instituts für Mathematik

Technische Universität Berlin 
The present paper is dedicated to the solution of constrained shape optimization problems by second order algorithms with respect to both, the primal and dual variables. This goal is realized by combining a Newton scheme for the primal variables with Mårtensson's concept of Lagrange multiplier functions for augmented Lagrangians.

AMS subject classification: 49Q10, 65N38, 90C90

Key words and phrases. Shape optimization, boundary element method, multiscale methods, augmented Lagrangian approach, Newton method, Mårtensson's approach. 


\title{
SECOND ORDER LAGRANGE MULTIPLIER APPROXIMATION FOR CONSTRAINED SHAPE OPTIMIZATION PROBLEMS
}

\author{
K. EPPLER AND HELMUT HARBRECHT
}

\begin{abstract}
The present paper is dedicated to the solution of constrained shape optimization problems by second order algorithms with respect to both, the primal and dual variables. This goal is realized by combining a Newton scheme for the primal variables with Måtensson's concept of Lagrange multiplier functions for augmented Lagrangians.
\end{abstract}

\section{INTRODUCTION}

Shape optimization is quite indispensable for designing and constructing industrial components. Many problems that arises in application, particularly in structural mechanics and in the optimal control of distributed parameter systems, can be formulated as the minimization of functionals defined over a class of admissible domains. Therefore, such problems have been intensively studied in the literature throughout the last 25-30 years (see [16, 17], [21, 23], and the references therein). From the practical as well as from the theoretical point of view, to prevent optimal shapes from degeneration, several constraints have to be taken into account. More recently, the computation of the related dual variables, i.e. the Lagrange multipliers, becomes of increasing interest due to applicational and theoretical reasons. On the one hand, there are several applications, where these multipliers have an important physical meaning like in the electromagnetic shaping of liquid metals (see e.g., [19, $20])$. On the other hand, their computation is important by itself for the investigation of sufficient optimality conditions in shape optimization [6].

In $[9,11]$ we developed first and second order optimization algorithms for a class of elliptic shape optimization problems, where a powerful wavelet BEM is proposed for the computation of the state and related quantities. Additional equality and/or inequality constraints of functional type are treated by an augmented Lagrangian technique, which turns out to be more stable and efficient than a penalty method. In particular, by the standard update procedure, a linear convergence is provided

Date: September 30, 2003.

Key words and phrases. Shape optimization, boundary element method, multiscale methods, augmented Lagrangian approach, Newton method, Mårtensson's approach. 
for the Lagrange multipliers. However, due to the efficiency of the Newton method with respect to the primal variables, that is the shape respective its finite dimensional approximation, this slow convergence of the Lagrange multipliers becomes in our opinion the bootleneck of the over-all algorithm. Furthermore, the question of a faster approximation of the dual variables is important as we explained above. Following an idea of Mårtensson [18], second order convergence is realizable, if only active equality constraints are present. Due to known degeneration tendencies in shape problems, exactly this situation occurs very often in practical and theoretical shape optimization problems. Consequently, the goal of the present paper is to demonstrate this approach for the class of shape problems considered in [9]. Of course, both the standard method and its modification are independent of the underlying shape calculus and of the numerical method for solving the state equation.

The outline of the present paper is as follows. In the first section we briefly introduce our setup for solving the considered shape optimization problems developed in $[9$, 10, 11]. The second section is dedicated to the augmented Lagrangian approach and the improvement of Mårtensson by introducing so-called Lagrange multiplier functions [18]. The last section presents the numerical results.

\section{Shape optimization}

1.1. The model problem. Let $\Upsilon$ denote the set of all bounded domains $\Omega \in C^{2, \alpha}$, $\Omega \subseteq D \subseteq \mathbb{R}^{2}$, starshaped with respect to $B_{\delta}(\mathbf{0})$. The outer security set $D \subseteq \mathbb{R}^{2}$ (the hold all set) is simply connected and closed, but not necessarily bounded. Setting $\Gamma:=\partial \Omega$, we consider the following shape problem:

$$
J(\Omega)=\int_{\Omega} h(\mathbf{x}) u(\mathbf{x})+h_{0}(\mathbf{x}) d \mathbf{x}=\min _{\Omega \in \Upsilon}
$$

subject to

$$
\begin{aligned}
J_{1}(\Omega) & =\int_{\Omega} h_{1}(\mathbf{x}) d \mathbf{x}=c_{1}, \\
J_{2}(\Omega) & =\int_{\Omega}^{\Omega} h_{2}(\mathbf{x}) d \mathbf{x}=c_{2}, \\
& \vdots \\
J_{m}(\Omega) & =\int_{\Omega} h_{m}(\mathbf{x}) d \mathbf{x}=c_{m},
\end{aligned}
$$

where the state function $u$ solves the Dirichlet boundary value problem

$$
\begin{aligned}
-\Delta u=f & \text { in } \Omega, \\
u=g & \text { on } \Gamma .
\end{aligned}
$$


In order to conceive a well posed problem the functions $f, g, h$ and $h_{0}, \ldots, h_{n}$ are assumed sufficiently regular on the whole set $D$. Of course, constraints of the type

$$
J_{i}(\Omega)=\int_{\Gamma} h_{i}(\mathbf{x}) d \sigma_{\mathbf{x}}
$$

may be considered in our setup as well. Constraints of practical interest in shape problems are the volume of the domain $\int_{\Omega} 1 d \mathbf{x}$ or its perimeter $\int_{\Gamma} 1 d \sigma_{\mathbf{x}}$, for example.

Let us remark that (1.1) includes also the important case of energy functionals. Consider the state equation having homogeneous boundary conditions $(g \equiv 0)$, then partial integration yields

$$
J(\Omega)=\int_{\Omega}\|\nabla u(\mathbf{x})\|^{2} d \mathbf{x}=\int_{\Omega} f u d \mathbf{x}
$$

that is $h \equiv f$ and $h_{0} \equiv 0$.

1.2. Shape calculus. Clearly, the starshaped domain $\Omega \in \Upsilon$ can be identified with a function which describes its boundary, i.e., in polar coordinates we have

$$
\Gamma:=\left\{\gamma(\phi)=r(\phi)\left[\begin{array}{c}
\cos \phi \\
\sin \phi
\end{array}\right]: \phi \in[0,2 \pi]\right\},
$$

where $r \in C_{\text {per }}^{2, \alpha}[0,2 \pi]$ is a positive function with $r>\delta$ and

$$
C_{\text {per }}^{2, \alpha}[0,2 \pi]=\left\{r \in C^{2, \alpha}[0,2 \pi]: r^{(i)}(0)=r^{(i)}(2 \pi), i=0,1,2\right\} .
$$

As a standard variation for perturbed domains $\Omega_{\varepsilon}$ and boundaries $\Gamma_{\varepsilon}$, respectively, we introduce a function $d r \in C_{\text {per }}^{2, \alpha}[0,2 \pi]$

$$
r_{\varepsilon}(\phi)=r(\phi)+\varepsilon d r(\phi),
$$

where $\gamma_{\varepsilon}(\phi)=r_{\varepsilon}(\phi) \mathbf{e}_{r}(\phi)$ is always a Jordan curve. Herein, $\mathbf{e}_{r}(\phi)=[\cos \phi, \sin \phi]^{T}$ denotes the unit vector in the outer radial direction. The main advantage of this simple approach is a complete embedding of the shape problem into a Banach space setting. That is, both the shapes and its increments, can be viewed as elements of $C_{\text {per }}^{2, \alpha}[0,2 \pi]$.

Let us remark that boundary variation is an appropriate ansatz since first and second order directional derivatives can be represented by boundary integrals, cf. [7, 8].

1.3. BIE formulation and fast solvers. It turns out, that introducing the adjoint state $p$ by

$$
\begin{aligned}
-\Delta p=h & \text { in } \Omega, \\
p=0 & \text { on } \Gamma,
\end{aligned}
$$

the directional derivative $\nabla J(\Omega)[d r]$ requires the knowledge of $\partial u / \partial \mathbf{n}$ and $\partial p / \partial \mathbf{n}$, where the state function $u$ satisfies (1.3), cf. [9]. 
In addition, defining the local shape derivative $d u[d r]$ and the adjoint local shape derivative $d p[d r]$ via

$$
\begin{aligned}
\Delta d u[d r]=0 & \text { in } \Omega, \\
d u[d r]=d r\left\langle\mathbf{e}_{r}, \mathbf{n}\right\rangle \frac{\partial(g-u)}{\partial \mathbf{n}} & \text { on } \Gamma,
\end{aligned}
$$

and

$$
\begin{aligned}
\Delta d p[d r] & =0 & & \text { in } \Omega, \\
d p[d r] & =-d r\left\langle\mathbf{e}_{r}, \mathbf{n}\right\rangle \frac{\partial p}{\partial \mathbf{n}} & & \text { on } \Gamma,
\end{aligned}
$$

respectively, the computation of $\nabla^{2} J(\Omega)\left[d r_{1}, d r_{2}\right]$ requires the quantities $\partial^{2} u /(\partial \mathbf{n} \partial \mathbf{t})$, $\partial^{2} p /(\partial \mathbf{n} \partial \mathbf{t}), \partial d u[d r] / \partial \mathbf{n}$, and $\partial d p[d r] / \partial \mathbf{n}$, see [9] for details.

As shown in $[9,11]$, employing suitable Newton potentials, the computation of the derivatives of $u, d u$, and the associated adjoints can be performed efficiently by boundary integral equations (BIEs). Boundary element methods provide a common tool for the solution of such equations. In general, cardinal B-splines are used as ansatz functions in the Galerkin formulation. However, these traditional discretizations yield densely populated and in general ill conditioned system matrices. Hence, a numerical scheme is of at least quadratic complexity.

The crucial idea of the wavelet Galerkin scheme is a change of bases, i.e., applying appropriate wavelet bases instead of the traditional single-scale bases. On the one hand, based on the well known norm equivalences of wavelet bases, the diagonals of the system matrices define optimal preconditioners, cf. [3, 5, 22]. On the other hand, the resulting quasi-sparse system matrices can be compressed without loss of accuracy such that the complexity for the solution of the boundary integral equations becomes linear, cf. [4, 13, 14, 15].

1.4. Boundary approximation. Since the infinite dimensional optimization problem cannot be solved directly, we replace it by an finite dimensional problem. Based on the polar coordinate approach, we can express the smooth function $r \in$ $C_{\text {per }}^{2, \alpha}([0,2 \pi])$ by the Fourier series

$$
r(\phi)=a_{0}+\sum_{n=1}^{\infty} a_{n} \cos n \phi+a_{-n} \sin n \phi .
$$

Hence, it is reasonable to take the truncated Fourier series

$$
r_{N}(\phi)=a_{0}+\sum_{n=1}^{N} a_{n} \cos n \phi+a_{-n} \sin n \phi .
$$


as approximation of $r$. We mention that also other boundary representations like Bsplines can be considered as well. The advantages of our approach is an exponential convergence $r_{N} \rightarrow r$ if the shape is analytical.

To this end, since $r_{N}$ has the $2 N+1$ degrees of freedom $a_{-N}, a_{1-N}, \ldots, a_{N}$, we replace the infinite dimensional set of admissible domains $\Upsilon$ by an $2 N+1$ dimensional set $\Upsilon_{N}$ defined by

$$
\Upsilon_{N}:=\left\{a_{-N}, a_{1-N}, \ldots, a_{N} \in \mathbb{R}: r_{N}(\phi)>0, \phi \in[0,2 \pi]\right\} \subset \mathbb{R}^{2 N+1},
$$

Consequently, according to this finite dimensional setting, we conclude

$$
\nabla J(\Omega)=\left[\begin{array}{c}
\nabla J(\Omega)[\cos N \phi] \\
\nabla J(\Omega)[\cos (N-1) \phi] \\
\vdots \\
\nabla J(\Omega)[\sin N \phi],
\end{array}\right] \in \mathbb{R}^{2 N+1}
$$

and likewise for the shape hessian $\nabla^{2} J(\Omega) \in \mathbb{R}^{(2 N+1) \times(2 N+1)}$.

\section{Optimization of CONSTRAined PROBlEMS}

2.1. Augmented Lagrangian functionals. Setting $\boldsymbol{\lambda}=\left(\lambda_{1}, \lambda_{2} \ldots, \lambda_{m}\right)^{T} \in \mathbb{R}^{m}$, the minimization problem defined by (1.1) and (1.2) implies to find the stationary point $\left(\Omega^{\star}, \boldsymbol{\lambda}^{\star}\right) \in \Upsilon_{N} \times \mathbb{R}^{m}$ of the following modified augmented Lagrangian functional

$$
L_{\alpha}(\Omega, \boldsymbol{\lambda})=J(\Omega)+\boldsymbol{\lambda}^{T}\left[\begin{array}{c}
C_{1}(\Omega)-c_{1} \\
C_{2}(\Omega)-c_{2} \\
\vdots \\
C_{m}(\Omega)-c_{m}
\end{array}\right]+\frac{\alpha}{2}\left\|\left[\begin{array}{c}
C_{1}(\Omega)-c_{1} \\
C_{2}(\Omega)-c_{2} \\
\vdots \\
C_{m}(\Omega)-c_{m}
\end{array}\right]\right\|^{2}
$$

Of course, the choice $\alpha=0$ yields the pure Lagrangian while $\boldsymbol{\lambda}=\mathbf{0}$ and $\alpha \rightarrow \infty$ is known as standard quadratic penalty method. However, both methods have some drawbacks from the numerical point of view, cf. $[12,18]$, for example.

In order to avoid these difficulties, we consider $\alpha>0$ fixed but appropriately chosen and perform the following standard augmented Lagrangian algorithm:

- initialization: choose initial guesses $\boldsymbol{\lambda}^{(0)}$ for $\boldsymbol{\lambda}^{\star}$ and $\Omega^{(0)}$ and $\Omega^{\star}$,

- inner iteration: solve $L_{\alpha}\left(\Omega, \boldsymbol{\lambda}^{(n)}\right)=\min$ with initial guess $\Omega^{(n)}$,

- outer iteration: update

$$
\boldsymbol{\lambda}^{(n+1)}:=\boldsymbol{\lambda}^{(n)}-\alpha\left[\begin{array}{c}
C_{1}(\Omega)-c_{1} \\
C_{2}(\Omega)-c_{2} \\
\vdots \\
C_{m}(\Omega)-c_{m}
\end{array}\right]
$$

and choose $\Omega^{(n+1)}$ as the solution from the inner iteration.

As we have observed in [9], a Newton scheme for minimizing $L_{\alpha}\left(\Omega, \boldsymbol{\lambda}^{(n)}\right)$ makes a line-search nearly obsolete in comparison to first order methods. As a consequence, 
the bottleneck during the iteration process is now the slow first order approach of the Lagrange multipliers according to the following theorem, cf. [2, 12].

Theorem 2.1. Assuming that the minimization problem (1.1) and (1.2) has a solution $\Omega^{\star} \in \Upsilon_{N}$ with $\boldsymbol{\lambda}^{\star} \in \mathbb{R}^{m}$ satisfying the KKT-conditions as well as the sufficient second order conditions, then the update rule (2.6) leads a linear convergence $\boldsymbol{\lambda}^{(n)} \rightarrow \boldsymbol{\lambda}^{\star}$, provided that the initial guesses are properly chosen.

We like to mention that augmented Lagrangian techniques are also available for inequality constraints, but due to the lack of smoothness second order algorithms would not work.

The gradient of the functional (2.5) reads as

$$
\nabla L_{\alpha}(\Omega, \boldsymbol{\lambda})=\nabla J(\Omega)+\sum_{i=1}^{m}\left(\lambda_{i}+\alpha\left(C_{i}(\Omega)-c_{i}\right)\right) \nabla C_{i}(\Omega)
$$

and its hessian as

$$
\begin{aligned}
\nabla^{2} L_{\alpha}(\Omega, \boldsymbol{\lambda}) & =\nabla^{2} J(\Omega)+\sum_{i=1}^{m}\left(\lambda_{i}+\alpha\left(C_{i}(\Omega)-c_{i}\right)\right) \nabla^{2} C_{i}(\Omega) \\
& +\alpha\left[\begin{array}{c}
\nabla C_{1}(\Omega) \\
\nabla C_{2}(\Omega) \\
\vdots \\
\nabla C_{m}(\Omega)
\end{array}\right]^{T}\left[\begin{array}{c}
\nabla C_{1}(\Omega) \\
\nabla C_{2}(\Omega) \\
\vdots \\
\nabla C_{m}(\Omega)
\end{array}\right]
\end{aligned}
$$

2.2. Second order Lagrange multiplier update. Mårtensson [18] proposed a second order method for the Lagrange multiplier. More precisely, the multiplier is seen as a function depending on the primal variable, i.e., $\boldsymbol{\lambda}=\boldsymbol{\lambda}(\Omega)$, namely

$$
\boldsymbol{\lambda}(\Omega)=-\mathbf{A}(\Omega)^{-1}\left[\begin{array}{c}
\nabla^{T} C_{1}(\Omega) \nabla J(\Omega) \\
\nabla^{T} C_{2}(\Omega) \nabla J(\Omega) \\
\vdots \\
\nabla^{T} C_{m}(\Omega) \nabla J(\Omega)
\end{array}\right] \in \mathbb{R}^{m},
$$

where the matrix $\mathbf{A}(\Omega)$ is given by

$$
\mathbf{A}(\Omega)=\left[\begin{array}{cccc}
\nabla^{T} C_{1}(\Omega) \nabla C_{1}(\Omega) & \ldots & \nabla^{T} C_{1}(\Omega) \nabla C_{m}(\Omega) \\
\nabla^{T} C_{2}(\Omega) \nabla C_{1}(\Omega) & \ldots & \nabla^{T} C_{2}(\Omega) \nabla C_{m}(\Omega) \\
\vdots & & \vdots \\
\nabla^{T} C_{m}(\Omega) \nabla C_{1}(\Omega) & \ldots & \nabla^{T} C_{m}(\Omega) \nabla C_{m}(\Omega)
\end{array}\right] \in \mathbb{R}^{m \times m}
$$

Instead of the constant multiplier in the shape functional (2.5), a first order Taylor expansion of the above multiplier function, developed at the given initial guess $\Omega^{(n)}$, 
is inserted into the augmented Lagrangian functional. Consequently, using

$$
\begin{aligned}
\boldsymbol{\lambda}^{\prime}(\Omega) & =-\mathbf{A}(\Omega)^{-1}\left\{\left[\begin{array}{c}
\nabla^{T} C_{1}(\Omega) \nabla^{2} J(\Omega)+\nabla^{T} J(\Omega) \nabla^{2} C_{1}(\Omega) \\
\nabla^{T} C_{2}(\Omega) \nabla^{2} J(\Omega)+\nabla^{T} J(\Omega) \nabla^{2} C_{2}(\Omega) \\
\vdots \\
\nabla^{T} C_{m}(\Omega) \nabla^{2} J(\Omega)+\nabla^{T} J(\Omega) \nabla^{2} C_{n}(\Omega)
\end{array}\right]\right. \\
& \left.-\sum_{i=1}^{m} \lambda_{i}\left[\begin{array}{c}
\nabla^{T} C_{1}(\Omega) \nabla^{2} C_{i}(\Omega)+\nabla^{T} C_{i}(\Omega) \nabla^{2} C_{1}(\Omega) \\
\nabla^{T} C_{2}(\Omega) \nabla^{2} C_{i}(\Omega)+\nabla^{T} C_{i}(\Omega) \nabla^{2} C_{2}(\Omega) \\
\vdots \\
\nabla^{T} C_{m}(\Omega) \nabla^{2} C_{i}(\Omega)+\nabla^{T} C_{i}(\Omega) \nabla^{2} C_{m}(\Omega)
\end{array}\right]\right\} \in \mathbb{R}^{m \times(2 N+1)},
\end{aligned}
$$

we consider the functional

$$
L_{\alpha}^{(n)}(\Omega)=L_{\alpha}\left(\Omega, \boldsymbol{\lambda}\left(\Omega^{(n)}\right)+\boldsymbol{\lambda}^{\prime}\left(\Omega^{(n)}\right)\left(\Omega-\Omega^{(n)}\right)\right)
$$

Herein, the difference $\Omega-\Omega^{(n)}$ has to be understood as the difference of the associated Fourier coefficients. Of course, the gradient and the hessian of (2.8) are slightly different compared to those of (2.5), but the computation is straightforward and all ingredients appear also in the first and second order derivatives of (2.5): the gradient is given by

$$
\begin{aligned}
\nabla L_{\alpha}^{(n)}(\Omega) & =\nabla L_{\alpha}\left(\Omega, \boldsymbol{\lambda}\left(\Omega^{(n)}\right)+\boldsymbol{\lambda}^{\prime}\left(\Omega^{(n)}\right)\left(\Omega-\Omega^{(n)}\right)\right) \\
& +\boldsymbol{\lambda}^{\prime}\left(\Omega^{(n)}\right)^{T}\left[\begin{array}{c}
C_{1}(\Omega)-c_{1} \\
C_{2}(\Omega)-c_{2} \\
\vdots \\
C_{m}(\Omega)-c_{m}
\end{array}\right]
\end{aligned}
$$

and the hessian by

$$
\begin{aligned}
\nabla^{2} L_{\alpha}^{(n)}(\Omega) & =\nabla^{2} L_{\alpha}\left(\Omega, \boldsymbol{\lambda}\left(\Omega^{(n)}\right)+\boldsymbol{\lambda}^{\prime}\left(\Omega^{(n)}\right)\left(\Omega-\Omega^{(n)}\right)\right) \\
& +\boldsymbol{\lambda}^{\prime}\left(\Omega^{(n)}\right)^{T}\left[\begin{array}{c}
\nabla^{T} C_{1}(\Omega) \\
\nabla^{T} C_{2}(\Omega) \\
\vdots \\
\nabla^{T} C_{m}(\Omega)
\end{array}\right]+\left[\begin{array}{c}
\nabla^{T} C_{1}(\Omega) \\
\nabla^{T} C_{2}(\Omega) \\
\vdots \\
\nabla^{T} C_{m}(\Omega)
\end{array}\right] \boldsymbol{\lambda}^{\prime}\left(\Omega^{(n)}\right) .
\end{aligned}
$$

Mårtensson's approach yields the following optimization algorithm:

- initialization: choose an initial guess $\Omega^{(0)}$ for $\Omega^{\star}$

- inner iteration: solve $L_{\alpha}^{(n)}(\Omega)=\min$ by the Newton scheme with initial guess $\Omega^{(n)}$,

- outer iteration: choose $\Omega^{(n+1)}$ as the solution from the inner iteration.

Theorem 2.2 (Mårtensson [18]). Assume $\alpha$ sufficiently large and $\left(\Omega^{\star}, \boldsymbol{\lambda}^{\star}\right) \in \Upsilon_{N} \times$ $\mathbb{R}^{m}$ satisfying the assertion of theorem 2.1, then the above algorithm converges quadratically to $\left(\Omega^{\star}, \boldsymbol{\lambda}^{\star}\right)$, i.e., this algorithm has one step convergence for linearquadratic problems. 


\section{Numerical RESUlts}

3.1. A shape problem from planar elasticity. For comparison reasons we employ first a model problem where the optimal shape and the dual variables are known analytically. We consider a cylindric circular bar which is homogeneous and isotropic with a planar, simply connected cross section $\Omega \in \mathbb{R}^{2}$. We follow Banichuk and Karihaloo [1] but normalize the shear modulus $G=1$ and the elastic modulus $E=1$. We want to solve the problem of maximizing the torsional rigidity of the bar subject to given equality constraints on the stiffness rigidity and the volume.

First, we briefly recall the mathematical formulation of the quantities. The torsional rigidity is calculated by

$$
J(\Omega)=2 \int_{\Omega} u(\mathbf{x}) d \mathbf{x}
$$

Thus, since we want to maximize the torsional rigidity, we have $h \equiv-2$ and $h_{0} \equiv 0$ in (1.1). The stress function $u=u(\Omega)$ satisfies

$$
\begin{aligned}
-\Delta u=2 & \text { in } \Omega, \\
u=0 & \text { on } \Gamma .
\end{aligned}
$$

The bending rigidity with respect to a fixed barycentre in the origin is given by

$$
J_{1}(\Omega)=\int_{\Omega} y^{2} d \mathbf{x}
$$

The volume of the domain and its (simplified) barycentre coordinates read as

$$
J_{2}(\Omega)=\int_{\Omega} d \mathbf{x}, \quad J_{3}(\Omega)=\int_{\Omega} x d \mathbf{x}, \quad J_{4}(\Omega)=\int_{\Omega} y d \mathbf{x} .
$$

Choosing $c_{1}=\sqrt{2} \pi / 4, c_{2}=\pi$, and $c_{3}=c_{4}=0$, then the optimal shape is the ellipse with semiaxes $h_{x}=2^{-1 / 4}$ and $h_{y}=2^{1 / 4}$. The associated Lagrange multipliers are $\lambda_{1}=-4 / 9, \lambda_{2}=8 \sqrt{2} / 9$, and $\lambda_{3}=\lambda_{4}=0$, cf. [1].

The numerical setting is as follows. We approximate the boundary via 33 Fourier coefficients, that is the choice $N=16$ according to (1.4). The boundary integral equations are discretized using 256 boundary elements. The penalty parameter $\alpha$ is set to 10. In the inner iteration we use a Newton scheme, see [9] for the details. The outer iteration is started with the unit circle.

In figure 3.1 we compare produced errors of Mårtensson's method (solid line) with that of the traditional update rule (2.6) using different initial guesses for the Lagrange multipliers, namely

- $(0,1,0,0)^{T}$ produced from Mårtensson's formula (2.7) at the unit circle (dashed line),

- $(-1,1,0,0)^{T}$, that is $\lambda_{i}^{(0)}=\operatorname{sgn} \lambda_{i}^{\star}$, 

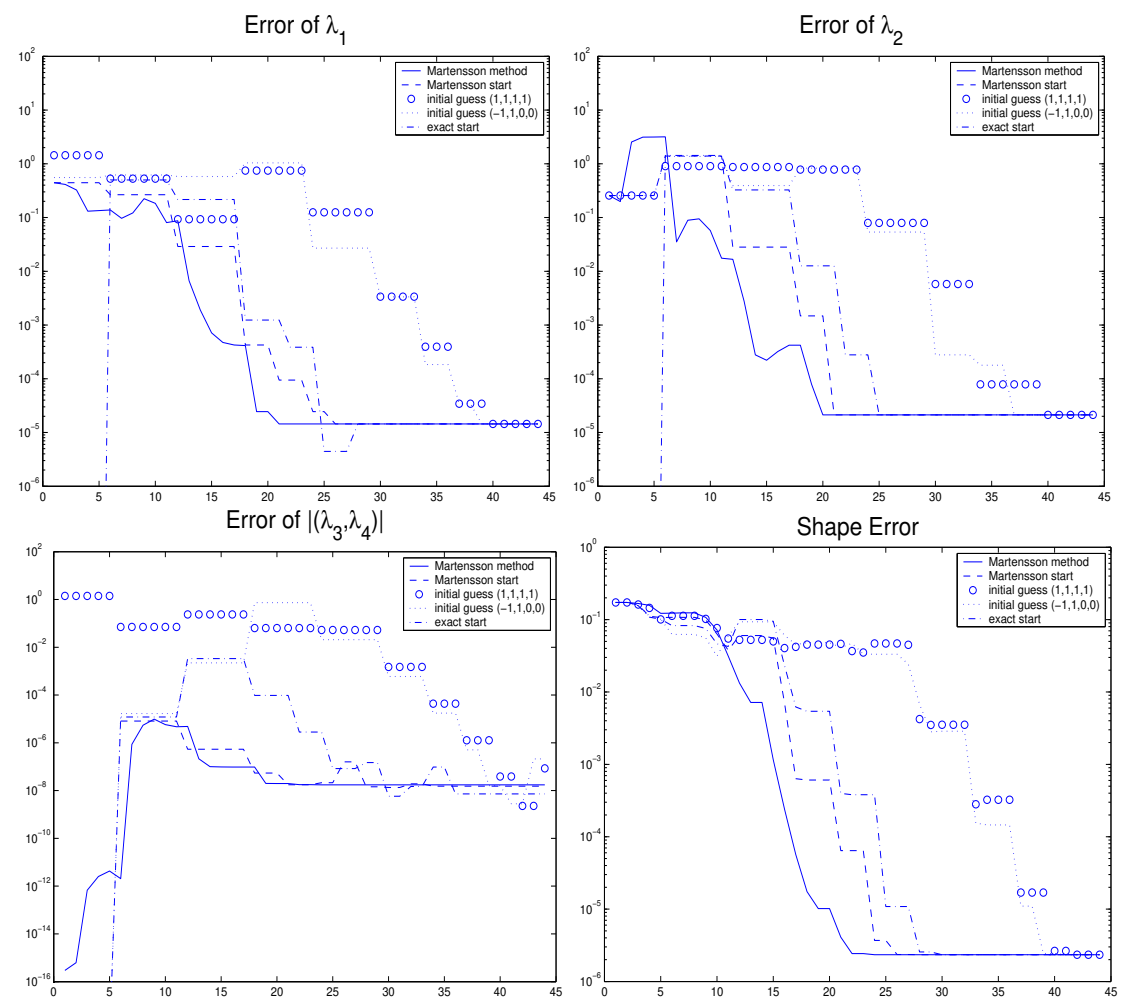

FiguRE 3.1. Errors of the dual variables and the shape during the iteration.

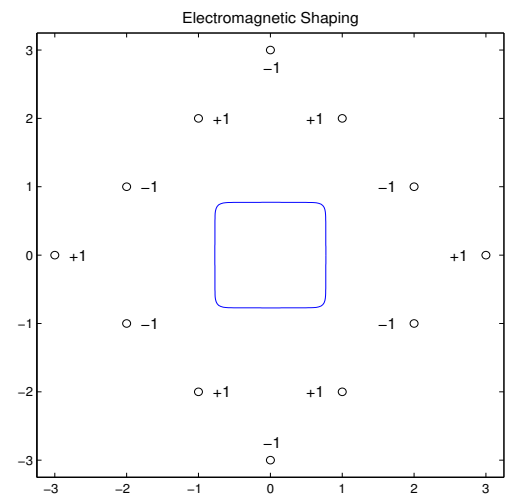

Figure 3.2. Configuration of electromagnets and liquid metal.

- $(1,1,1,1)^{T}$ (indicated by circles), and

- $\boldsymbol{\lambda}^{\star}$ (dash-dotted line).

As one figures out, Mårtensson's method yields the best convergence while the best traditional scheme uses the initial guess computed by $(2.7)$.

3.2. Exterior electromagnetic shaping. We consider a cylindric vertical column of molten liquid metal with planar, simply connected cross section falling down 
in an electromagnetic field generated by vertical conductors of radii $\varepsilon>0$. The frequency of the imposed current is very high such that electromagnetic forces are reduced to the magnetic pressure acting on the interface. In the equilibrium case, a stationary horizontal cross section $\Omega^{c}=\mathbb{R}^{2} \backslash \Omega$ of fixed volume $c_{1}$ arises and the three dimensional problem reduces to the following two dimensional shape problem: Seek $\Omega$ such that

$$
J(\Omega)=-\int_{\Omega}\|\nabla u\|^{2} d \mathbf{x}+A \int_{\Gamma} 1 d \sigma=\min ,
$$

where

$$
\begin{aligned}
-\Delta u & =j:=\sum_{i=1}^{M} \frac{\alpha_{i}}{\pi \varepsilon^{2}} \chi_{B_{\varepsilon}\left(\mathbf{x}_{i}\right)} & & \text { in } \Omega, \\
u & =0 & & \text { on } \Gamma, \\
u & =\mathcal{O}(1) & & \text { as }\|\mathbf{x}\| \rightarrow \infty \\
\nabla u & =\mathcal{O}\left(\|\mathbf{x}\|^{-2}\right) & & \text { as }\|\mathbf{x}\| \rightarrow \infty .
\end{aligned}
$$

subject to $J_{1}(\Omega)=\int_{\Omega^{c}} 1 d \mathbf{x}=c_{1}$. We emphasize that the Lagrange multiplier $\lambda_{1}$ corresponds to the magnetic preassure on the surface of the liquid metal.

We choose a $A=0.001, c_{1}=3 \pi / 4$, and twelve conductors in the positions and with amperage $\alpha_{i}$ in accordance with figure 3.2. The choice of $\varepsilon>0$ does not influence the solution, cf. [10]. We use 65 Fourier coefficients for the boundary, i.e. $N=32$, 512 boundary elements, and $\alpha=5$. We use the circle of volume $c_{1}$ as initial guess for the Newton scheme. Mårtensson's method finishes the iteration in 110.38 secs. after 10 Newton steps while the traditional scheme (with initial guess $\lambda_{1}^{(0)}=0$ ) requires 192.55 secs. and 25 Newton steps. Both schemes produce the same $\lambda_{1}^{\star}=-0.0101458$.

\section{REFERENCES}

[1] N.V. Banichuk and B.L. Karihaloo. Minimum-weight design of multi-purpose cylindrical bars. International Journal of solids and Structures, 12:267-273, 1976.

[2] D.P. Bertsekas. Constrained optimization and Lagrange multiplier methods. Academic Press, New York, 1982.

[3] W. Dahmen. Wavelet and multiscale methods for operator equations. Acta Numerica, 6:55-228, 1997.

[4] W. Dahmen, H. Harbrecht, and R. Schneider. Compression techniques for boundary integral equations - optimal complexity estimates. Technical Report SFB 393/0206, TU Chemnitz, Institute of Mathematics, D-09107 Chemnitz, 2002. submitted for publication.

[5] W. Dahmen and A. Kunoth. Multilevel preconditioning. Numer. Math., 63:315-344, 1992.

[6] M. Dambrine and M. Pierre. About stability of equilibrium shapes. M2AN, 34:811$834,2000$. 
[7] K. Eppler. Boundary integral representations of second derivatives in shape optimization. Discussiones Mathematicae (Differential Inclusion Control and Optimization), 20:63-78, 2000.

[8] K. Eppler. Optimal shape design for elliptic equations via BIE-methods. J. of Applied Mathematics and Computer Science, 10:487-516, 2000.

[9] K. Eppler and H. Harbrecht. 2nd order shape optimization using wavelet BEM. Technical Report 06-2003, TU Berlin, Institute of Mathematics, Str. d. 17. Juni, D-10623 Berlin, 2003. submitted for publication.

[10] K. Eppler and H. Harbrecht. Exterior electromagnetic shaping using wavelet BEM. Technical Report 13-2003, TU Berlin, Institute of Mathematics, Str. d. 17. Juni, D10623 Berlin, 2003. submitted for publication.

[11] K. Eppler and H. Harbrecht. Numerical solution of elliptic shape optimization problems using wavelet-based BEM. Optim. Methods Softw., 18:105-123, 2003.

[12] Ch. Grossmann and J. Terno. Numerik der Optimierung. B.G. Teubner, Stuttgart, 1993.

[13] H. Harbrecht, F. Paiva, C. Pérez, and R. Schneider. Biorthogonal wavelet approximation for the coupling of FEM-BEM. Numer. Math., 92:325-356, 2002.

[14] H. Harbrecht, F. Paiva, C. Pérez, and R. Schneider. Wavelet preconditioning for the coupling of FEM-BEM. Numerical Linear Algebra with Applications, 10:197-222, 2003.

[15] H. Harbrecht and R. Schneider. Wavelet Galerkin Schemes for 2D-BEM. In Operator Theory: Advances and Applications, volume 121. Birkhäuser, 2001.

[16] J. Haslinger and P. Neitaanmäki. Finite element approximation for optimal shape, material and topology design, 2nd edition. Wiley, Chichester, 1996.

[17] A.M. Khludnev and J. Sokolowski. Modelling and control in solid mechanics. Birkhäuser, Basel, 1997.

[18] K. Mårtensson. A new approach to constrained function optimization. J. of Optimization Theory and Application, 12:531-554, 1973.

[19] A. Novruzi and J.-R. Roche. Second derivatives, newton method, application to shape optimization. Technical Report No. 2555, INRIA, Nancy, France, 1995.

[20] M. Pierre and J.-R. Roche. Computation of free surfaces in the electromagnetic shaping of liquid metals by optimization algorithms. Eur. J. Mech, B/Fluids, 10:489-500, 1991.

[21] O. Pironneau. Optimal shape design for elliptic systems. Springer, New York, 1983.

[22] R. Schneider. Multiskalen- und Wavelet-Matrixkompression: Analysisbasierte Methoden zur Lösung großer vollbesetzter Gleichungssysteme. B.G. Teubner, Stuttgart, 1998.

[23] J. Sokolowski and J.-P. Zolesio. Introduction to shape optimization. Springer, Berlin, 1992. 
TU Berlin, Institute of Mathematics, Str. D. 17. Juni, 10623 Berlin, GerMANY.

E-mail address: eppler@math.tu-berlin.de

TU Chemnitz, Faculty of Mathematics, 09107 Chemnitz, Germany

E-mail address: helmut.harbrecht@mathematik.tu-chemnitz.de 